Meta

Journal des traducteurs

Translators' Journal

\title{
Propriétés transformationnelles unaires en lexicographie informatique
}

\section{Xavier Blanco}

Volume 55, numéro 1, mars 2010

Le parcours du sens : d'une langue à l'autre — Mélanges offerts à André Clas

The Way of Meaning: From a Language to Another - Collection of Articles Offered to André Clas

URI : https://id.erudit.org/iderudit/039601ar

DOI : https://doi.org/10.7202/039601ar

Aller au sommaire du numéro

\section{Éditeur(s)}

Les Presses de l'Université de Montréal

ISSN

0026-0452 (imprimé)

1492-1421 (numérique)

Découvrir la revue

Citer cet article

Blanco, X. (2010). Propriétés transformationnelles unaires en lexicographie informatique. Meta, 55(1), 42-57. https://doi.org/10.7202/039601ar

\section{Résumé de l'article}

Le présent article fait état d'un inventaire des propriétés transformationnelles unaires pensé pour le codage des prédicats dans un dictionnaire électronique conçu principalement pour la traduction automatique espagnol-français. Le travail a un caractère éminemment métalexicographique et appliqué. Il ne contient pas de nouveautés théoriques puisque toutes les transformations présentées sont bien connues. Son but est de fournir à un linguiste informaticien travaillant en contexte multilingue une vue d'ensemble de cette partie de la description linguistique. En amont des propriétés transformationnelles, nous traitons les alternances de traits syntactico-sémantiques pour une position argumentale donnée, les alternances de prépositions, les interprétations non agentives et les diathèses spéciales. Comme transformations unaires, nous présentons : l'insertion de déterminants nominaux, l'insertion de partie appropriée, les insertions d'un argument, les pronominalisations, l'effacement d'un argument, les permutations d'arguments (sous-divisées en : permutations proprement dites, détachements, extrapositions et extractions), les changements de diathèse (la passive pleine, la passive indirecte, le réfléchi, la passive impersonnelle et différents types de restructurations : les restructurations $\mathrm{N}_{0}-$ (Prép) $\mathrm{N}_{1}$, les restructurations $\mathrm{N}_{0}$ Prép $\mathrm{N}_{2}$ et les restructurations (Prép) $\mathrm{N}_{1}^{0}$ - Prép $\mathrm{N}_{2}$ ). Finalement, nous abordons les variantes syntaxiques des verbes support. Les propriétés retenues sont illustrées moyennant des exemples en français et en espagnol.
Ce document est protégé par la loi sur le droit d'auteur. L'utilisation des services d’Érudit (y compris la reproduction) est assujettie à sa politique d'utilisation que vous pouvez consulter en ligne.

https://apropos.erudit.org/fr/usagers/politique-dutilisation/ 


\title{
Propriétés transformationnelles unaires en lexicographie informatique
}

\author{
XAVIER BLANCO \\ Universitat Autònoma de Barcelona, Barcelone, Espagne \\ xavier.blanco@uab.cat
}

\section{RÉSUMÉ}

Le présent article fait état d'un inventaire des propriétés transformationnelles unaires pensé pour le codage des prédicats dans un dictionnaire électronique conçu principalement pour la traduction automatique espagnol-français. Le travail a un caractère éminemment métalexicographique et appliqué. II ne contient pas de nouveautés théoriques puisque toutes les transformations présentées sont bien connues. Son but est de fournir à un linguiste informaticien travaillant en contexte multilingue une vue d'ensemble de cette partie de la description linguistique. En amont des propriétés transformationnelles, nous traitons les alternances de traits syntactico-sémantiques pour une position argumentale donnée, les alternances de prépositions, les interprétations non agentives et les diathèses spéciales. Comme transformations unaires, nous présentons: l'insertion de déterminants nominaux, l'insertion de partie appropriée, les insertions d'un argument, les pronominalisations, l'effacement d'un argument, les permutations d'arguments (sousdivisées en: permutations proprement dites, détachements, extrapositions et extractions), les changements de diathèse (la passive pleine, la passive indirecte, le réfléchi, la passive impersonnelle et différents types de restructurations: les restructurations $\mathrm{N}_{0}-$ (Prép) $N_{1}$, les restructurations $N_{0}-$ Prép $N_{2}$ et les restructurations (Prép) $N_{1}-$ Prép $N_{2}$ ). Finalement, nous abordons les variantes syntaxiques des verbes support. Les propriétés retenues sont illustrées moyennant des exemples en français et en espagnol.

\section{ABSTRACT}

This paper presents an inventory of unary transformational properties intended to encode predicates in an electronic dictionary. This work has mainly a metalexicographical and applied character. The paper does not present any theoretical innovation, since all the discussed transformations are well known. The aim of the paper is to provide the computational linguist working in a multilingual context with an overview of this aspect of the linguistic description. First, as a related phenomenon, we present the alternation of syntactico-semantic features, the preposition alternations, the non-agentive interpretations and the special diathesis. As unary transformations we present: insertion of nominal determiners, insertion of appropriate part, insertion of one argument, pronominalisation, deletion of an argument, permutation of arguments (subdivided in permutations per se, frontings, extrapositions and extractions), diathesis change (i.e., full passive, indirect passive, reflexive, impersonal passive and different kinds of restructurations: $\mathrm{N}_{0}-$ (Prép) $N_{1}$ restructurations, $N_{0}$ - Prép $N_{2}$ restructurations and (Prép) $N_{1}$ - Prép $N_{2}$ restructurations). Finally, we introduce the syntactic variants for support verbs. The discussed properties are exemplified by means of French and Spanish sentences.

\section{MOTS-CLÉS/KEYWORDS}

dictionnaire électronique, propriété transformationnelle, prédicat unaire, schéma d'arguments, traduction français-espagnol

electronic dictionary, transformational feature, unary predicate, argument scheme, French-Spanish translation 
Le présent article fait état d'un ensemble des propriétés transformationnelles unaires que nous avons choisi de prendre en compte pour le codage des prédicats dans un dictionnaire électronique conçu principalement pour la traduction automatique espagnol-français (Blanco 2001). Afin de ne pas alourdir notre présentation, nous illustrerons les propriétés retenues tantôt avec des exemples en français, tantôt en espagnol, en signalant les éventuelles divergences entre les deux langues.

Ce travail a un caractère éminemment métalexicographique et appliqué. Il ne contient pas de nouveautés théoriques puisque toutes les transformations présentées sont bien connues. Son but est de fournir à un linguiste informaticien travaillant en contexte multilingue une première vue d'ensemble de ce pan de la description linguistique.

Nos observations et nos exemples s'inspirent, d'une part, des travaux de Harris (1964/1981, 1965/1981) et du lexique-grammaire (Boons, Guillet et al. 1976; Gross 1975; Guillet et Leclère 1992; Leclère 1990, entre autres) et, d'autre part, de la théorie Sens-Texte (Mel'čuk 1992; 1993). Soulignons que les recherches et l'enseignement de Gaston Gross ont eu une influence décisive sur notre façon de comprendre la linguistique et d'intégrer les apports de différents courants de pensée. Cet article lui doit donc beaucoup. Bien entendu, nous rendons hommage également à l'enseignement et à la personnalité d'André Clas, auquel cet article est dédié.

Notons que cette présentation, nécessairement très schématique, exclut d'emblée des questions importantes comme l'application successive de plusieurs transformations et, en conséquence, l'ordre et les contraintes qui existent dans leur combinatoire. Il va sans dire que, pour tenir compte de ces questions, il faut se doter au préalable d'une liste opérationnelle de transformations. Nous espérons que cet article puisse contribuer en partie à cela.

\section{Concepts de base}

La discussion sur les propriétés transformationnelles que nous allons entamer exige le recours systématique à quelques concepts-définitions de base ${ }^{1}$ (que nous présentons en italique):

1) Les propriétés transformationnelles sont formulées par rapport à des prédicats. Les transformations unaires ne concernent qu'un prédicat. Les transformations binaires concernent deux prédicats (et, par extension, plus de deux);

2) Un prédicat a des actants sémantiques, des actants syntaxiques profonds et des actants syntaxiques de surface;

3) Un actant sémantique d'un prédicat est un argument, au sens logique, dudit prédi$\mathrm{cat}^{2}$. Il correspond à une variable dans la représentation sémantique du prédicat. Il est représenté par les symboles I, II, III, IV... La représentation correspondant aux actants sémantiques d'un prédicat est le schéma d'arguments, par exemple, apprivoiser (Nhumain, Nanimal), planter (Nhumain, Nvégétal)... Les actants représentés dans un schéma d'arguments sont étiquetés moyennant des traits syntactico-sémantiques (par exemple, humain, abstrait...) et des classes d'objets (par exemple, <vêtements>, <crimes>...) (Gross 1994; Le Pesant et Mathieu-Colas 1998);

4) Un actant syntaxique profond d'un prédicat est un syntagme qui exprime au niveau syntaxique profond un des actants sémantiques du prédicat. Il est représenté par les symboles $\mathrm{N}_{0}, \mathrm{~N}_{1}, \mathrm{~N}_{2}, \mathrm{~N}_{3} \ldots$ Ces actants présentent les propriétés classiques que 
l'on reconnaît au sujet, à l'objet direct, à l'objet indirect... Ainsi, le $\mathrm{N}_{0}$ régit l'accord verbal, le $\mathrm{N}_{1}$ devient le sujet de la voix passive, etc. La représentation non marquée correspondant aux actants syntaxiques profonds est la structure de base, par exemple, $\mathrm{N}_{0} \mathrm{~V} / \mathrm{N}_{0} \mathrm{~V} \mathrm{~N}_{1} / \mathrm{N}_{0} \mathrm{~V}$ Prép $\mathrm{N}_{1} \ldots$;

5) Un actant syntaxique de surface est un syntagme représenté par des mots-formes effectivement produits dans une phrase donnée et soumis à linéarisation (donc avec un ordre fixé dans la phrase), par exemple, $\mathrm{N}_{1}$ Vpassif Prép $\mathrm{N}_{0}$ (La lettre a été écrite par $L u c)$;

6) Une phrase canonique est une phrase où il y a une symétrie parfaite entre actants sémantiques, actants syntaxiques profonds et actants syntaxiques de surface, par exemple, Luc donne une pomme à Max;

7) Une phrase transformée est une phrase où cette symétrie n'est pas respectée, que ce soit au niveau des actants syntaxiques profonds (Une pomme a été donnée à Max par Luc) ou au niveau syntaxique de surface (à Max, Luc a donné une pomme; Luc, il donne une pomme à Max);

8) La non-symétrie d'une phrase transformée par rapport à une phrase canonique est due principalement à:

- Un changement d'ordre dans la linéarisation de la phrase (permutation d'actants);

- La modification de la forme d'un actant (par exemple, par pronominalisation, par effacement...);

- La modification de la forme d'un prédicat (par exemple, par coordination, par ajout de modalité);

- L'insertion d'un nouvel actant ou d'un élément qui prend la place, en surface, d'un actant.

9) Une entrée de dictionnaire électronique comporte des informations sur la structure de base associée à un prédicat. À partir de cette structure de base, il est possible de générer une phrase canonique en appliquant des règles générales. Par contre, la génération des phrases transformées ne peut pas être prise en charge par de telles règles. Des champs spécifiques du dictionnaire doivent être prévus afin de rendre compte de la possibilité / impossibilité, pour un prédicat donné, d’apparaître dans tel type de phrase transformée. Une discussion sur les propriétés transformationnelles est, dans ce sens, une discussion sur des catégories de microstructure d'un dictionnaire électronique et sur leurs valeurs.

Comme il a été signalé ci-dessus, nous partons de la base que l'on dispose de dictionnaires électroniques qui contiennent une grande quantité de prédicats accompagnés de leurs schémas d'arguments caractéristiques. Les schémas d'arguments représentent les actants sémantiques d'un prédicat. Ces schémas sont généralisés, c'est-à-dire que les arguments sont étiquetés moyennant des outils de représentation distributionnelle comme les traits syntactico-sémantiques (humain, inanimé, concret, abstrait...) et les classes d'objets et de prédicats (<vêtements $>$, <nourritures $>$, $<$ délits $>$, <étendues d'eau >...).

Nous avons donc, d'une part, un schéma d'arguments généralisé correspondant à une phrase élémentaire et représentant les actants sémantiques, par exemple:

(1) manger (Nhum, Ninc $<$ nourritures $>$ )

et, d'autre part, une structure de base pour ledit schéma représentant les actants syntaxiques profonds du prédicat décrit:

(2) $\mathrm{N}_{0} \mathrm{~V} \mathrm{~N}_{1}$ 
Si nous maintenons la symétrie entre ces actants syntaxiques profonds et les actants syntaxiques de surface, qui en donnent la linéarisation, nous pouvons considérer que le système est capable de générer un ensemble de suites grammaticales possibles en base à des consultations de dictionnaires. Cela implique encore l'instantiation de Nhum et de Ninc < nourritures $>$ à des unités lexicales et l'actualisation du schéma moyennant la conjugaison, la flexion et la détermination:

(3) Luc mange un chocolat.

Luc mange du riz.

Luc mange un steak frites.

On dispose ainsi d'une série de phrases canoniques. Or, il est clair que l'ensemble ainsi généré est loin de couvrir la totalité des phrases grammaticales observables ${ }^{3}$ (par exemple, C'est du riz que Luc mange).

Le problème est double. D'une part, il existe un ensemble de suites observables qui ne correspondent pas aux structures de base et dont il faut faire l'inventaire (par exemple, À cette question correspond cette réponse). D'autre part, bien que ces structures soient formalisables $\left(\mathrm{N}_{0} \mathrm{~V}\right.$ Prép $\mathrm{N}_{1}=$ Prép $\left.\mathrm{N}_{1} \mathrm{~V} \mathrm{~N}_{0}\right)$ elles ne sont pas pour autant généralisables. Ainsi, nous avons $\mathrm{N}_{0} \mathrm{~V}$ Prép $\mathrm{N}_{1}$ (Luc pense à Max), mais ${ }^{\star} \grave{A}$ Max pense Luc. Cette deuxième question est cruciale en traitement automatique des langues où bien évidemment, il ne suffit pas de décrire formellement une propriété, il faut aussi en donner l'extension exacte.

Notre but pourrait être alors reformulé comme suit: quelles sont les propriétés linguistiques d'un prédicat donné qu'il faut coder dans un dictionnaire électronique pour prévoir des linéarisations autres que celle qui est considérée comme canonique pour ce prédicat? Nous appellerons ces propriétés des propriétés transformationnelles, dans la mesure où il s'agit de propriétés combinatoires de l'unité lexicale qui ne peuvent pas être codées dans la zone strictement distributionnelle de l'article lexicographique ${ }^{4}$.

Étant donné que nous nous occupons de l'élaboration de dictionnaires, nous souhaiterions formuler ces questions sous forme d'un cahier de charges ou d'un mode d'emploi pour le codage de propriétés transformationnelles dans un dictionnaire électronique. Cet article se veut une première ébauche d'un tel travail.

\section{En amont des propriétés transformationnelles}

La structure de base associée à un prédicat donné peut présenter des alternances. Dans la mesure où deux structures de base ne sont pas possibles simultanément, on est en présence de plus d'une chaîne graphique et, en conséquence, d'une relation entre des chaînes, sans qu'il semble pour autant justifié de parler de transformation.

\subsection{Alternances de traits syntactico-sémantiques pour une position argumentale}

Certains prédicats admettent de modifier, par métonymie, le trait syntactico-sémantique de certains arguments. Ainsi, on a un changement de locatif à humain collectif dans: 
(4) Luc a diffusé le match en Espagne.

Luc a diffusé le match aux Espagnols.

Ces cas sont, pour l'instant, marqués par disjonction de traits dans les schémas d'arguments des prédicats concernés. Des généralisations intéressantes pourraient éventuellement en faciliter le codage (Le Pesant 2000).

\subsection{Alternances de prépositions}

La préposition marqueur d'argument peut avoir des variantes possibles ou être optionnelle:

(5) Luc s'est battu avec Max.

Luc s'est battu contre Max.

Luc habló de este problema.

Luc habló sobre este problema.

Il suffit, pour ces cas, de marquer la disjonction de préposition au sein de la structure de base. Il va sans dire que cette alternance ne doit être confondue ni avec les cas de prépositions différentes pour des verbes homonymes,

(6) Luc reparó los desperfectos. (= réparer les dégâts)

Luc reparó en los desperfectos. (= remarquer les dégâts)

ni avec l'alternance d'une préposition introduisant un complément locatif, qui correspond à l'application de la fonction lexicale Loc (Mel'čuk 1996):

(7) Luc habite $a u$ Japon.

Luc habite en France.

Luc habite cette maison.

Luc habite dans cette maison.

\subsection{Interprétations non agentives}

Certains prédicats admettent une double interprétation agentive/non agentive du $\mathrm{N}_{0}{ }^{5}$ :

(8) Luc s'est coupé au doigt (accidentellement + intentionnellement).

Luc asustó a Max (sin querer + a propósito).

L'adverbe est, en fait, pléonastique par rapport à une des interprétations, mais il la sélectionne de façon non ambiguë. Il est possible que l'existence d'un $\mathrm{N}_{2}$ permette de sélectionner une des interprétations: Luc asusta a Max con esos gritos est, en géneral, interprété comme non agentif. Notons, en plus, que la passive Max fue asustado por Luc, qui tend à sélectionner l'interprétation agentive, n'accepte pas le $\mathrm{N}_{2}$ : *Max fue asustado por Luc con esos gritos.

\subsection{Diathèses spéciales}

Dans la plupart des cas, il existe une symétrie possible entre les actants sémantiques et les actants syntaxiques profonds du prédicat. Notons, cependant, des cas comme plaire, où nous avons le schéma d'arguments plaire (Nhum, Nnr) et des phrases canoniques comme Ce film plaît à $L u c$, où $\mathrm{N}_{0}=$ II (deuxième actant sémantique) et 
$\mathrm{N}_{1}=\mathrm{I}$ (premier actant sémantique). C'est le cas aussi de l'espagnol gustar ${ }^{6}$. Ces prédicats présentent une sorte de non-canonicité foncière. Ainsi, Luc pense à Max est une phrase canonique alors que Ce film plaît à Luc n'en est pas une, car la symétrie entre actants sémantiques et syntaxiques est écartée d'emblée.

Certains auteurs parlent aussi de diathèse spéciale pour faire référence à des phénomènes comme la présence obligatoire d'un adverbe:

(9) Luc se porte bien. ${ }^{\star}$ Luc se porte.

ou la négation obligatoire:

(10) No cabe duda de $Q u$ ${ }^{*}$ Cabe duda de $\mathrm{Qu}^{7}$

Dans ce cas, il n'y a qu'une suite canonique possible. Dans d'autres cas, comme les adverbes à polarité, on peut en avoir deux:

(11) Cette viande sent (bon + mauvais).

Cette viande sent. $=$ Cette viande sent mauvais .

Nous ne pensons pas que ces derniers cas concernent la diathèse, étant donné que celle-ci ne touche que la relation entre actants sémantiques et actants syntaxiques profonds. Nous préférons donc traiter ces cas comme des verbes composés. Ainsi, par exemple, encontrarse bien (se porter bien) est dans le dictionnaire des verbes composés de la classe CADV (complément adverbial obligatoire) et no caber duda de $\mathrm{Qu}$ - dans la classe à négation obligatoire (Gross 1982). Évidemment, il reste à rendre compte des variations paradigmatiques (Luc se porte comme un charme). Nous pensons qu'il s'agit là de la présence obligatoire de la fonction lexicale Bon appliquée au prédicat. Pour sentir, nous aurions obligatoirement soit Bon, soit AntiBon, avec effacement approprié possible pour AntiBon, mais pas pour Bon.

\section{Transformations unaires}

Rappelons que les transformations unaires sont celles qui s'appliquent à un seul prédicat.

\subsection{Insertion de déterminants nominaux}

Lactualisation peut comporter l'insertion de déterminants nominaux. Du point de vue du traitement automatique, il est important que ces noms soient analysés comme déterminants et aucunement comme têtes sémantiques du syntagme, sous peine de ne pas reconnaître le schéma d'arguments existant dans la phrase. En tant que déterminants, ces noms concernent la quantification, la notion d'ensemble et d'élément et aussi, quand ils sont associés à des noms prédicatifs, l'intensité et certaines données aspectuelles (fréquentatif, itératif...):

(12) Luc a bu trois bières.

Luc a bu (trois bouteilles + toute une caisse) de bières.

(13) Los turistas bajaron del autocar.

(Una avalancha + un grupo) de turistas bajó del autocar ${ }^{8}$. 
À notre avis, nous avons le même phénomène dans:

(14) Luc a engraissé le parterre (de bégonias).

Luc a engraissé les bégonias.

même si dans ce cas on pourrait penser que engraisser s'applique en fait à un locatif. En tout cas, le fait d'élargir à ces cas l'approche par déterminants nominaux permet de simplifier beaucoup la description lexicographique (Blanco 2002).

\subsection{Insertion de partie appropriée}

L'insertion d'un $\mathrm{N}$ dans une position argumentale donnée peut correspondre aussi à une relation méronymique / holonymique:

(15) Luc toucha Max.

Luc toucha l'épaule de Max.

Luc bouge.

Luc bouge les jambes.

Luc acarició al perro.

Luc acarició el lomo del perro9.

La partie appropriée peut aussi être insérée comme $\mathrm{N}_{2}$ :

(16) Luc dispose les livres sur le rayonnage.

Luc dispose les livres sur la tranche. (Guillet et Leclère 1992: 416)

$\mathrm{Au}$ sens large, on pourrait classer sous cette rubrique des relations comme:

(17) Luc libère Max de ses préoccupations.

Luc libère l'esprit de Max de ses préoccupations.

La notion de partie appropriée peut concerner aussi des ensembles fonctionnels, surtout en position de $\mathrm{N}_{0}$ :

(18) La voiture ralentit.

Le conducteur de la voiture ralentit.

La Banque de France a décidé de signer cet accord.

Les directeurs de la Banque de France ont décidé de signer cet accord.

Dans la pratique, nous avons traité provisoirement ce dernier problème en déclarant des sous-classes de noms humains correspondant à des moyens de transport, des institutions, etc.

Notons que les parties inaliénables sont appropriées mais que les parties appropriées ne sont pas nécessairement inaliénables. Ainsi les bras de Luc sont nécessairement $L u c$, mais le conducteur de la voiture n'est pas la voiture. Celle-ci a, par ailleurs, une syntaxe d'inanimé concret comme Banque de France a une syntaxe de locatif (Max a nettoyé le bras de Luc = Max a nettoyé Luc; Max a nettoyé la voiture de Luc $\neq$ Max a nettoyé Luc).

Il existe toutefois des prédicats dont les contraintes de sélection exigent une partie du corps et excluent un humain (par exemple, amputer). Ainsi, la relation:

(19) Luc s'est coupé.

Luc s'est coupé un bras. 
est, à notre avis, une fausse relation. Il y a deux unités lexicales sous couper: l'une est synonyme de amputer, l'autre non. Ainsi, Luc s'est coupé à un bras (Luc s'est coupé, par effacement de Prép $\mathrm{N}_{1} \mathrm{pc}$ ) s'oppose sémantiquement à Luc s'est coupé un bras. Observons que, une fois que Luc s'est coupé un bras, il ne peut plus se couper au bras. En tout cas, il peut couper ce bras. Autrement dit, cet inanimé concret n'est plus une partie du corps et n'a donc plus une syntaxe de partie inaliénable. Remarquons aussi que l'ajout du $\mathrm{N}_{1}=\mathrm{Npc}$ 'implique pas la disparition du se coréférent au $\mathrm{N}_{0}$ : Luc s'est coupé lui-même au bras. Mais si le bras n'est pas à lui: ${ }^{*}$ Luc s'est coupé Max au bras; Luc a coupé Max au bras.

\subsection{Insertion d'un argument}

Dans le dictionnaire, nous suivons le principe d'extension maximale des schémas d'arguments. Tout prédicat est décrit avec le nombre maximal de ses actants sémantiques, indépendamment de leur concrétion syntaxique et bien évidemment, du fait qu'il est effaçable ou pas. Après tout, le fait de marquer un argument donné comme effaçable implique de l'avoir reconnu préalablement comme tel (voir la section 3.5).

Cependant, il y a des arguments tellement pléonastiques qu'il semble plus raisonnable de les considérer comme des insertions possibles. Il s'agit notamment des objets internes :

(20) Luc dort.

${ }^{\star}$ Luc dort un sommeil.

Luc dort d'un sommeil léger ${ }^{10}$.

Les objets internes peuvent être aussi des $\mathrm{N}_{2}$ :

(21) Luc cultiva maíz.

Luc cultiva maíz en sus cultivos.

Nous trouvons aussi des réflexifs pléonastiques par rapport au $\mathrm{N}_{0}$ :

(22) Luc emporta les livres.

Luc emporta les livres avec lui.

et par rapport au $\mathrm{N}_{1}$ :

(23) Luc ha enrollado la cuerda.

Luc ha enrollado la cuerda sobre sí misma.

\subsection{Pronominalisations}

Nous donnons différents types de pronominalisations sans commentaires, dans la mesure où elles sont bien connues et relativement faciles à représenter:

(24) Luc a posé le vase sur la table.

Il l'a posé sur la table.

Luc l'a posé sur la table.

Luc y a posé le vase.

(25) Luc a dépouillé Max de ses biens.

Il a dépouillé Max de ses biens.

Luc l'a dépouillé de ses biens.

Luc en a dépouillé Max. 
(26) Luc plaît à Max.

Luc lui obéit $v{ }^{*}$ Luc obéit à lui.

Luc pense à Max.

Luc pense à lui $v s{ }^{\star}$ Luc lui pense.

(27) Luc consacre de l'argent à son appartement.

Luc (lui + y) consacre de l'argent.

(28) Luc sait que Max est parti.

Luc le sait $v s$ Luc sait $(c e l a+$ ça).

Luc aime que tu viennes.

${ }^{\star}$ Luc l'aime $v s$ Luc aime ceci.

(29) Luc veille à ce que tout soit rangé.

Luc y veille.

(30) Luc reconnaît Max à ce qu'il est toujours en colère. Luc reconnaît (là Max + Max à cela).

(31) Luc se réjouit de ce que tu sois heureux. Luc s'en réjouit.

Notons aussi la pronominalisation contrastive (avec intonation intensive):

(32) Luc est parti. Lui est parti.

\subsection{Effacement d'un argument}

Un argument peut être effacé si le contexte le fait redondant (par exemple, en parlant d'un gâteau dans une recette):

(33) Cuire au four pendant 20 minutes.

Un argument peut être effacé en motivant une lecture du verbe comme propriété caractéristique du $\mathrm{N}_{0}$ (emploi absolu):

(34) Ce chien mord les gens.

Ce chien mord.

Ce couteau coupe les objets.

Ce couteau coupe.

Remarquons que, si l'on applique la négation, la lecture de propriété caractéristique est accentuée. Ainsi, ce chien ne mord pas ne veut pas dire qu'il soit incapable de mordre (des aliments, par exemple), mais qu'il n'agresse pas. Ce couteau ne coupe pas n'implique pas qu'on ne soit pas en train de couper quelque chose avec le couteau en question, mais qu'il n'est pas suffisamment aiguisé. Nous sommes plutôt partisans de traiter ces emplois des formes mordre et couper comme des unités lexicales à part dans la mesure où elles sont sémantiquement proches des adjectifs.

Évidemment, nous avons des cas douteux comme:

(35) Ce film choque toujours les gens.

Ce film choque.

Le fait qu'on ait: 
(36) Ce film a choqué Luc, mais il n'est pas choquant.

?Ce film choque toujours tout le monde, mais il n'est pas choquant.

fait qu'on hésite à traiter $\mathrm{N}_{0}$ choquer comme un effacement de $\mathrm{N}_{0}$ choquer $\mathrm{Nhum}$ ou comme une autre unité lexicale (bien qu'intimement liée à la précédente). Pour l'instant, nous penchons pour la première option à cause du nombre élevé de verbes ayant un tel comportement.

Un argument peut aussi être tout simplement effacé avec perte de l'information correspondante:

(37) Luc mange une pomme.

Luc mange.

À ce moment-là, une des interprétations possibles est l'interprétation d'argument généralisé (Luc mange des aliments). Mais d'autres possibilités sont à considérer: Luc mange beaucoup, Luc mange bien, Luc a un bon coup de fourchette, Luc est en train de prendre son repas (par exemple, Luc est allé manger sera en général interprété comme Luc prend son repas, et difficilement comme Luc est allé manger un chocolat).

En conséquence, le fait d'indiquer le caractère sous-spécifiable d'un argument devrait s'accompagner de l'interprétation possible de cette sous-spécification, par exemple, Luc conduit = Luc est en train de conduire (une voiture) ou Luc a son permis. En effet, on peut avoir Luc n'a pas son permis mais il conduit tandis que?Luc n'a pas son permis et il conduit, ce qui est une preuve a contrario que l'interprétation 'avoir son permis' est parfaitement possible. Cette précision est indispensable pour qu'un système ait la capacité de reconstruire un schéma d'arguments pertinent. Une piste possible pour optimiser la notation de cette propriété serait de la mettre en rapport avec les noms d'agent possibles (Luc conduit $=$ Luc est un conducteur $)^{11}$.

Les $\mathrm{N}_{2}$ et $\mathrm{N}_{3}$ (de distance, d'instrument, d'origine, de destination...) peuvent aussi être effacés:

(38) Luc a acheminé les colis sur $1000 \mathrm{~km}$.

Luc a acheminé les colis.

(39) Luc balaie sa chambre avec un balai.

Luc balaie sa chambre.

(40) Luc a transformé le fichier de HTML en PDF.

Luc a transformé le fichier en PDF.

${ }^{*}$ Luc a transformé le fichier de HTML (origine).

On a certains $\mathrm{N}_{4}$ (de somme d'argent, de lieu de passage...) qui seraient aussi effaçables (et même facilement effaçables):

(41) Luc a loué son appartement à Max pour un mois à $600 €$.

Luc a envoyé la lettre de Paris à Barcelone via Lyon.

\subsection{Permutations d'arguments}

Certaines transformations préservent le nombre d'arguments mais en changent la linéarisation. Ces transformations se différencient des changements de diathèse (voir section 3.7.) en ce qu'elles ne concernent que les actants syntaxiques de surface. 


\subsubsection{Permutations proprement dites}

Nous avons ainsi, des permutations $\mathrm{N}_{1}-\mathrm{N}_{2}$ :

(42) Luc vend une voiture à Max. Luc vend à Max une voiture.

Cette permutation s'explique souvent par des raisons stylistiques, par exemple quand le $\mathrm{N}_{1}$ est nettement plus long que le $\mathrm{N}_{2}$ :

(43) ?Luc a donné le stylo qu'il avait trouvé hier sur la table de son voisin à Max. Luc a donné à Max le stylo qu'il avait trouvé hier sur la table de son voisin.

La permutation peut aussi concerner l'attribut, une relation dite équative ${ }^{12}$ :

(44) Luc est le chef. Le chef est Luc.

ou le $\mathrm{N}_{0} \mathrm{~V}$ dans certaines complétives:

(45) Le travail que Luc fait est inutile. Le travail que fait Luc est inutile.

Nous avons ensuite les permutations de Prép $\mathrm{N}_{1}$ ou de Prép $\mathrm{N}_{2}$ par rapport au $\mathrm{N}_{0}$ :

Cette question correspond à cette réponse.

À cette réponse correspond cette question.

\subsubsection{Détachements}

Les détachements (appelés parfois aussi dislocations) du $\mathrm{N}_{0}$ ou du $\mathrm{N}_{1}$ impliquent une pronominalisation, une prosodie particulière (pause ou accent d'intensité) ou une permutation $\mathrm{V} \mathrm{N}_{0}$ :

(47) Luc ne réfléchit pas.

Qui ne réfléchit pas? Lúc ne réfléchit pas.

Luc, il ne réfléchit pas.

(48) Un certain nombre d'ennuis découlent de ta décision.

De ta décision, un certain nombre d'ennuis en découlent.

\subsubsection{Extrapositions}

Nous trouvons aussi des extrapositions en il ou il $y$ a. Les extrapositions impliquent l'apparition d'un argument supplémentaire, quoique sémantiquement vide:

(49) Faire ceci plaît à Luc.

Il plaît à Luc de faire ceci.

Ce qui plaît à Luc est de faire ceci.

(50) Un vase est sur la table.

Il y a un vase sur la table.

\subsubsection{Extractions}

Les extractions en c'est qui/que sont possibles pour n'importe quel argument $\left(\mathrm{N}_{0}, \mathrm{~N}_{1}\right.$, Prép $\mathrm{N}_{1}$...) et semblent accentuer le caractère contrastif de la permutation. On dirait que l'argument supplémentaire hésite à apparaître comme actant syntaxique profond (l'accord avec le V est hésitant). 
(51) Les filles sont parties.

Ce sont les filles qui sont parties.

C'est les filles qui sont parties.

\subsection{Changements de diathèse}

Les différents types de permutations supposent des changements d'ordre des actants syntaxiques de surface par rapport à des actants syntaxiques profonds. Cet alinéa, par contre, concerne les changements de diathèse, c'est-à-dire le changement d'ordre des actants syntaxiques profonds par rapport aux actants sémantiques d'un prédicat donné.

\subsubsection{Les passifs}

\subsubsection{La passive pleine}

Le deuxième actant sémantique peut occuper la position de premier actant syntaxique profond:

(52) Luc a coupé les roses.

Les roses ont été coupées (par Luc).

\subsubsection{La passive indirecte}

En anglais, nous avons la passive indirecte où le $\mathrm{N}_{2}$ passe à $\mathrm{N}_{0}$. Dans le dictionnaire espagnol-français, nous n'avons codé aucun cas.

(53) Luc gave an apple to Max.

Max was given an apple by Luc.

\subsubsection{Le réfléchi}

L'identification des premier et deuxième actants syntaxiques profonds constitue aussi un changement de diathèse:

(54) Luc peigne Max.

Luc se peigne.

\subsubsection{La passive impersonnelle}

Dans la passive impersonnelle, le premier actant syntaxique profond est supprimé. Laccord se fait alors avec le deuxième actant:

(55) Luc construye estas casas (aquí desde hace años). Se construyen estas casas (aquí desde hace años).

Cette construction est parfois appelée (à notre avis improprement) ergative. Le déplacement du $\mathrm{N}_{1}$ vers la gauche donne la suite (appelée parfois se moyen ${ }^{13}$ ):

(56) Estas casas se construyen (aquí desde hace años).

Notons aussi :

(57) Luc dijo muchas cosas.

Alguien dijo muchas cosas. (pronominalisation)

Se dijeron muchas cosas.

Muchas cosas se dijeron ${ }^{14}$. 
En français, nous avons les pronoms on, il.

(58) Luc construit des maisons.

On construit des maisons.

Il se construit des maisons.

Des maisons se contruisent ${ }^{15}$.

(59) Luc a dit cela.

On a dit cela. (pronominalisation)

Il s'est dit cela.

Cela s'est dit.

La pronominalisation par il s'applique parfois aussi à la structure de base $\mathrm{N}_{0} \mathrm{~V}$ Prép $\mathrm{N}_{1}$ :

(60) Luc a procédé à l'examen des malades.

Il a été procédé à l'examen des malades.

\subsubsection{Les restructurations}

Nous avons tendance à voir, dans les restructurations, une homonymie de deux unités lexicales à diathèses converses (comme acheter et vendre). En effet, d'abord, il n'y a pas ici de paradigme verbal passif ou moyen. Ensuite, les prépositions marqueurs d'arguments peuvent changer de façon imprévisible (surtout dans les restructurations de type $\mathrm{N}_{1}-$ Prép $\mathrm{N}_{2}$ ). Finalement, ce phénomène est clairement moins important, $\mathrm{du}$ point de vue quantitatif, que les passifs. Tout cela fait qu'il est beaucoup plus commode d'avoir tout simplement une autre entrée de dictionnaire pour chaque forme restructurée, avec la linéarisation correspondante et l'indication de l'unité lexicale converse dans la zone de combinatoire lexicale restreinte (fonction lexicale Conv).

\subsubsection{Restructurations $N_{0}-\left(\right.$ Prép) $N_{1}$}

Nous avons, d'abord, des restructurations avec permutation des premier et deuxième actants syntaxiques profonds (et des changements de Prép):

(61) Les étudiants fourmillent dans le couloir.

Le couloir fourmille d'étudiants.

\subsubsection{Restructurations $N_{0}-$ Prép $N_{2}$}

Nous trouvons aussi des cas où c'est le troisième actant sémantique qui passe à premier actant syntaxique profond (avec suppression du premier actant sémantique):

(62) Luc démontra son innocence grâce à la lettre.

La lettre démontra l'innocence (de Luc).

(63) Luc regroupe plusieurs documents dans chaque dossier. Chaque dossier regroupe plusieurs documents.

(64) Luc a fait un vin excellent avec ce raisin. Ce raisin fait un vin excellent. 


\subsubsection{Restructurations (Prép) $N_{1}-$ Prép $N_{2}$}

Nous avons aussi une permutation des deuxième et troisième actants syntaxiques profonds, accompagnée de changements de Prép:

(65) Luc grave ses initiales sur cette médaille.

Luc grave cette médaille de ses initiales.

\subsection{Les variantes syntaxiques des verbes support ${ }^{16}$}

Nous nous sommes centré jusqu'à présent sur les propriétés des phrases axées sur un prédicat verbal. Il ne faut cependant pas oublier les prédicats adjectivaux et nominaux. Par exemple, l'extraposition est possible pour les adjectifs:

(66) Qu’il pleuve est agréable à Luc.

Il est agréable à Luc qu'il pleuve.

Quand le nom est le noyau prédicatif d'une phrase, il est accompagné d'un verbe support. Dans la structure moins marquée, le nom prédicatif occupe la position de $\mathrm{N}_{1}$ et ses actants sémantiques les positions de $\mathrm{N}_{0}$ et $\mathrm{N}_{2}$ (par exemple, la première phrase de l'exemple 67). Mais le verbe support peut avoir des variantes à diathèse différente $^{17}$ (comme la deuxième phrase de l'exemple 67):

(67) Luc a donné un coup à Max. (Oper $\left.{ }_{1}\right)$ Max a reçu un coup de Luc. $\left(\right.$ Oper $\left._{2}\right)$

Cela nous met devant tout un système de paraphrases. En adoptant le système de fonctions lexicales proposé par Igor Mel'čuk (1992), nous aurons, entre autres relations possibles:

(68) Luc a fait une analyse détaillée de cette phrase. ( Oper $\left._{1}\right)$

Luc a soumis cette phrase à une analyse détaillée. ( Laborl $_{2}$ )

(69) Luc a donné cet ordre. (Oper $\left.{ }_{1}\right)$

Cet ordre provient de Luc. (Func $)_{1}$

(70) L’idée de partir en vacances est de Luc. (Func $)_{1}$

L'idée de Luc consiste à partir en vacances. $\left(\right.$ Func $\left._{2}\right)$

Bien évidemment, nous ne présentons que les paraphrases les plus fréquentes pour les langues considérées. D’autres paraphrases, bien que plus rares, sont théoriquement possibles.

\section{Conclusion}

Nous avons présenté un inventaire de propriétés transformationnelles unaires organisé de telle façon qu'il puisse être mis à profit pour l'élaboration de dictionnaires électroniques espagnol-français. Toutefois, d'autres propriétés seraient encore à envisager. Parmi les propriétés que nous souhaiterions replacer à l'intérieur du panorama ci-dessus, nous pourrions citer entre autres des relations comme Luc agrippe la branche vs Luc s'agrippe à la branche ou celle qui est mise en relief par l'exemple allemand über sein Benehmen urteilen vs sein Benehmen (accusatif) beurteilen. Par ailleurs, un deuxième article, organisé d'après les mêmes principes que celui-ci, sera consacré aux propriétés transformationnelles binaires. 


\section{NOTES}

1. Nous ne présentons que les concepts indispensables à la formulation de propriétés transformationnelles. Nous en donnons des définitions simplifiées et non formalisées.

2. Nous nous servirons dorénavant du terme argument comme synonyme de actant sémantique.

3. Même si l'on suppose que les dictionnaires sont complets et que les problèmes posés par l'actualisation et la linéarisation sont résolus, ce qui est loin d'être évident. L'élaboration et la maintenance de tels dictionnaires posent de sérieux problèmes (qui dépassent cependant l'objet de cet article). Quant à l'actualisation des phrases canoniques, d'importantes recherches restent à faire (par exemple pour ce qui est du rapport morphologie-sémantique, de la détermination des noms prédicatifs, etc.).

4. Parfois, c'est la relation entre deux distributions canoniques possibles qui est appelée transformationnelle, sans qu'il soit pour autant possible de préciser quelle est la transformée de l'autre. À la limite, on pourrait parler de distributions alternatives, mais dans la mesure où cela implique un rapport entre deux suites différentes, il s'agit déjà d’une information qui va au-delà de la distribution stricte.

5. Notons l'existence d'une paire comme lesionarse - autolesionarse en espagnol, qui permet de lever l'ambiguité: el preso se lesionó (suite ambiguë par rapport à l'agentivité du $\mathrm{N}_{0}$ ), el preso se autolesionó (suite non ambiguë).

6. Par rapport à like, où nous avons $\mathrm{N}_{0}=\mathrm{I}$ et $\mathrm{N}_{1}=\mathrm{II}$ : Luc likes this movie. Un autre exemple de diathèse spéciale serait l'adjectif allemand kalt (au sens 'avoir froid'): Es ist kalt mir.

7. Notons, pourtant, l'acceptabilité de la modalité interrogative: Qué duda cabe de Qu-.

8. Remarquons que les déterminants nominaux dépendent en même temps de l'argument sur lesquels ils s'appliquent et du prédicat: *Una avalancha de turistas estaba sentada en la estación.

9. On peut déplacer certains noms de partie du corps: Luc toucha Max sur l'épaule; Le soleil a bronzé Luc ( ${ }^{*}$ dans sa peau + aux bras); Luc acarició al perro en el lomo.

10. Dans le cas de ces objets internes qui correspondent à des noms abstraits (e.g. vivre une vie de $N$, vivre une vie Adj), il serait probablement préférable de traiter le $\mathrm{V}$ comme un verbe support, ce qui les rapprocherait de phrases comme Luc vit une (aventure + expérience). Il ne resterait qu’à déclarer le Modif obligatoire dans certaines combinatoires Vsup - Npréd pour rendre compte de l'agrammaticalité de la deuxième phrase de l'exemple 20.

11. Quand il s'agit de propriétés intrinsèques, il faut une prédication secondaire: Luc est un bon marcheur, ?Luc est un marcheur (Anscombre 2001).

12. Une permutation $\mathrm{N}_{0} \mathrm{~V} \mathrm{~N}_{1}-\mathrm{N}_{1} \mathrm{~V} \mathrm{~N}_{0}$ avec un verbe non attributif serait sentie comme une hyperbate et, donc, comme une figure de style.

13. Par exemple, Luc asa el pollo - El pollo se asa; Luc cuece la carne - La carne se cuece.

14. Notons la variante résultative dans: Luc dejó dichas muchas cosas - Se dejaron dichas muchas cosas - Muchas cosas (se dejaron + quedaron) dichas.

15. Notons aussi la possibilité, pour quelques prédicats: On a fait justice - Justice est faite.

16. Nous parlons de variantes syntaxiques des verbes support par opposition aux variantes sémantiques des verbes support, par exemple, variantes aspectuelles: Luc a le pouvoir - Luc (arrive au + prend le) pouvoir; variantes diastratiques: Luc m'a (donné + fichu) un coup; variantes intensives: Luc $(a+$ crève de) faim.

17. Évidemment, il est aussi possible d'appliquer la voix passive: Un coup lui a été donné par Max.

\section{RÉFÉRENCES}

Anscombre, Jean-Claude (2001): À propos des mécanismes sémantiques de formation de certains noms d'agent en français et en espagnol. Langages. 143:28-48.

Blanco, Xavier (2001): Dictionnaires électroniques et traduction automatique espagnol-français. Langages. 143:49-70.

Blanco, Xavier (2002): Les déterminants figés. Langages. 145:61-82.

Boons, Jean-Pierre, Guillet, Alain et LeClère, Christian (1976): La structure des phrases simples en français. Constructions intransitives. Genève: Droz.

Gross, Gaston (1994): Classes d'objets et description des verbes. Langages. 115:15-30.

Gross, Maurice (1975): Méthodes en syntaxe. Paris: Hermann.

Gross, Maurice (1982): Une classification des phrases figées du français. Revue québécoise de linguistique. 11(2):151-185. 
Guillet, Alain et Leclère, Christian (1992): La structure des phrases simples en français. Constructions transitives locatives. Genève: Droz.

HARris, Zellig S. (1964/1981): The Elementary Transformations. In: Henry Hiz, dir. Papers on Syntax. Dordrecht: D. Reidel, 211-235.

HARris, Zellig S. (1965/1981): Transformational Theory. In: Henry Hiz, dir. Papers on Syntax. Dordrecht: D. Reidel, 236-280.

Le Pesant, Denis et Mathieu-Colas, Michel (1998): Introduction aux classes d'objets. Langages. 131:6-33.

Le Pesant, Denis (2000): Six études de sémantique lexicale sur les noms locatifs. Mémoire d'habilitation non publié. Paris: Université Paris 13.

LECLÈre, Christian (1990): Organisation du lexique-grammaire des verbes français. Langue française. 87:112-122.

Mel'čuk, Igor (1992): Paraphrase et lexique: la théorie Sens-Texte. In: Igor Mel'čuk, Nadia Arbatchewsky-Jumarie, Lidija Iordanskaja, et al., dir. Dictionnaire explicatif et combinatoire du français contemporain III. Montréal: PUM, 9-58.

MeL'ČUK, Igor (1993): The inflectional category of voice: towards a more rigorous definition. In: Bernard Comrie et Maria Polinsky, dir. Causatives and Transitivity. Amsterdam: John Benjamins, 1-46.

Mel'Čuk, Igor (1996): Lexical Functions: A Tool for the Description of Lexical Relations in a Lexicon. In: Leo WANNER, dir. Lexical Functions in Lexicography and Natural Language Processing, Amsterdam: John Benjamins, 37-102. 